\title{
Population structure, inbreeding and local adaptation within an endangered riverine specialist: the nase (Chondrostoma nasus)
}

\author{
Alan G. Hudson · Pascal Vonlanthen • \\ Ole Seehausen
}

Received: 24 May 2013/Accepted: 1 March 2014/Published online: 6 April 2014

(C) Springer Science+Business Media Dordrecht 2014

\begin{abstract}
Chondrostoma nasus is a cyprinid fish with highly specialized, ecologically and geographically distinct, ontogenetic trophic niches. Nase population numbers across their Swiss range have shown massive declines and many localized extinctions. In this study, we integrate genetic data (AFLP, microsatellite, mtDNA sequence) with phenotypic and demographic analyses to survey patterns of neutral and adaptive genetic diversity in all extant (and one extinct) Swiss nase populations, with the aim to delineate intraspecific conservation units (CUs) and to inform future population management strategies. We discovered two major genetically and geographically distinct population groupings. The first population grouping comprises nase inhabiting rivers flowing into Lake Constance; the second comprises nase populations from Rhine drainages below Lake Constance. Within these clusters there is generally limited genetic differentiation among populations.
\end{abstract}

Alan G. Hudson and Pascal Vonlanthen contributed equally to this work.

Electronic supplementary material The online version of this article (doi:10.1007/s105920140590 3) contains supplementary material, which is available to authorized users.

A. G. Hudson · P. Vonlanthen · O. Seehausen Division of Aquatic Ecology, Institute of Ecology \& Evolution, University of Bern, Baltzerstrasse 6, 3012 Bern, Switzerland

A. G. Hudson · P. Vonlanthen · O. Seehausen Department of Fish Ecology and Evolution, EAWAG Center for Ecology, Evolution and Biogeochemistry, Seestrasse 79, 6047 Kastanienbaum, Switzerland

A. G. Hudson ( $\square)$

Department of Ecology and Environmental Science, Umeå

University, Linnaeus väg 6, 90187 Umeå, Sweden

e mail: alan.g.hudson@gmail.com
Genomic outlier scans based on 256 to 377 polymorphic AFLP loci revealed little evidence of local adaptation both within and among population clusters, with the exception of one candidate locus identified in scans involving the low genetic diversity Schanzengraben population. However, significant phenotypic differentiation in body shape between certain populations suggests a need for more intensive future studies of local adaptation. Our data strongly suggests that the two major population groups should be treated as distinct CUs, with any supplemental stocking and reintroductions sourced only from within the range of the CU concerned.

Keywords Chondrostoma nasus - Conservation genomics - Local adaptation - Population genomics . Outlier scans - Adaptive potential - Conservation units (CUs)

\section{Introduction}

Current species extinction rates are estimated to be 100 to 1,000 times the background level in the fossil record and are predicted to increase in severity (Mace and Purvis 2008). Rates of loss of intraspecific diversity are estimated to be at least an order of magnitude higher (Hughes et al. 1997). In this context, there is an increasingly urgent need to understand how neutral and adaptive genetic diversity is sequestered within and among the populations of declining species, and how distinct populations are adapted to and interact with their local environments. This knowledge is vital in implementing management that will effectively conserve current intraspecific diversity and ensure the adaptive potential of individual populations and the species as a whole in the face of changing selection regimes 
(Ashley et al. 2003; Stockwell et al. 2003; Mace and Purvis 2008; Funk et al. 2012). Through the study of patterns of geographical co-variation in areas of the genome affected by natural selection and those evolving under neutral processes, population units targeted for conservation effort can be better delineated (Crandall et al. 2000; Kohn et al. 2006; Allendorf et al. 2010; Funk et al. 2012). In turn, such study will also allow the identification of genomic regions involved in local adaptation and the adaptive phenotypic traits they underpin, greatly increasing our understanding of the factors controlling intraspecific diversity, population differentiation and persistence (Kohn et al. 2006; Allendorf et al. 2010; Ouborg et al. 2010).

One principal driver of intraspecific levels of neutral genetic diversity and how it is geographically structured in temperate fish species is climatic fluctuation during the Quaternary period (Bernatchez and Wilson 1998; Hewitt 2004). Cycles of isolation in glacial refugia have been interspersed by population expansion, and possible secondary contact and hybridization among refugial lineages during warm interglacial periods. Switzerland lies within the drainage basins of four of Europe's major river systems: the Rhine, Rhône, Danube and Po, providing multiple potential freshwater colonization routes into an area that was largely glaciated until 15,000 years ago. Accordingly, mtDNA phylogeographic studies have revealed varying patterns of intraspecific diversity among fish species inhabiting this area, both in the number of colonizing lineages and their degree of admixture (e.g. Durand et al. 1999; Nesbø et al. 1999; Bernatchez 2001; Brunner et al. 2001; Kotlík and Berrebi 2001; Salzburger et al. 2003, Barluenga et al. 2006; Hudson et al. 2011). Cytonuclear comparisons have also shown that whilst in some species mitochondrial and nuclear geographic patterns of native genetic diversity are largely congruent [e.g. Cottus gobio (Englbrecht et al. 2000; Vonlanthen et al. 2007)], other studies show major discordance between markers [e.g. Coregonus lavaretus complex (Hudson et al. 2011), Perca fluviatilis (Behrmann-Godel et al. 2004)]. Past glaciation could also indirectly affect the geographic structuring of adaptive genetic diversity. Variation in the colonization success of different freshwater fish species into postglacial regions could lead to reduced levels of interspecific competition/predation and potentially lead to increased ecological opportunity in colonized habitats and concomitant diversification for those species able to colonize (Schluter 1996; Taylor 1999).

Within intraspecific conservation units (CUs) reduction in the number, effective size and connectivity of the constituent populations will increase the overall threat of extinction. This increased extinction risk pertains not only to the potential for chance events to have a disproportionately negative effect on the effective population size, but also to the more insidious effects of genetic drift and increased mating between close relatives within declining populations.
In the short term these latter two processes could cause inbreeding depression in individuals and increase the overall genetic load of populations through the fixation of deleterious alleles, reducing overall population fitness (Willi et al. 2006; Kristensen et al. 2010). In the longer term, the decrease in standing genetic diversity through genetic drift could threaten future population viability by reducing the adaptive potential of populations to respond to novel selection pressures (Willi et al. 2006; Bouzat 2010; Ouborg et al. 2010). To counter these threats, genetic rescue/restoration approaches, involving the supplemental introduction of unrelated individuals into threatened wild populations, have often been proposed/implemented (Hedrick and Fredrickson 2010; Weeks et al. 2011). Genetic rescue approaches need to balance the benefits of increased genetic diversity with potential pitfalls such as the loss of unique intraspecific and cryptic interspecific diversity through genetic swamping and outbreeding depression (Edmands 2007; Weeks et al. 2011). The avoidance of these negative impacts necessitates detailed knowledge of the structuring of neutral and adaptive genetic diversity across the geographic range of the species concerned. In particular, to characterize the degree and ecological drivers of local adaptation, both in threatened (or indeed extinct) populations and in the potential source populations for (re)introductions (Stockwell et al. 2003; Frankham et al. 2011; Weeks et al. 2011; Funk et al. 2012).

In Switzerland, industrialization and increasing human population densities have had severe adverse effects on the major river systems. One species that has been particularly strongly impacted in its abundance and geographic distribution is the nase [Chondrostoma nasus (Linnaeus 1758)]. Nase are widely distributed in central and eastern Europe with drainages in the Rhine catchment (including those in Switzerland) forming the westernmost edge of its natural distribution (Kottelat and Freyhof 2007). Prior to industrialization, nase was one of the most common fish species in larger midland streams in Switzerland. However, population sizes have dramatically decreased over the last century. Many local populations are today extinct, and the remaining, populations are generally small and declining in numbers (Maier et al. 1995). Nase exhibit a complex life cycle with different life-stages specializing in distinct riverine niches. Adults generally live in relatively fast flowing and deep (one to two metres) sections of rivers with coarsely grained substrate, where they graze on periphyton on riverine rocks and stones (Dedual 1990; Huber and Kirchhofer 1998). To reproduce, nase migrate to upstream tributaries, sometimes over considerable distances, seeking out shallow riffles in strong currents to spawn (Penáz 1996; Huber and Kirchhofer 1998; Rakowitz et al. 2008). Like other juvenile leuciscines, newly hatched nase require slow flowing habitats and feed largely on rotifers. With increasing size, they gradually move into deeper and faster 
flowing waters in concert with a shift in diet to zooplankton, macro-invertebrates and detritus, until they finally specialize on their unique periphyton-feeding niche (Adamik and Obrdlik 1977; Huber and Kirchhofer 1998; Reckendorfer et al. 2001). They are a long-lived (up to 20 years) and relatively slow-maturing (three to seven years) species (Szabo 1958; Dedual 1990). As a consequence of this specialized and complex life cycle: fragmentation of rivers by dams and other obstructions, homogenization and loss of riverine habitats, pollution and artificial hydrological regimes in rivers have likely all played a major role in the nase's decline in Switzerland (Kirchhofer 1996).

The only phylogeographic study of European C. nasus to date used allozymes to reveal a high degree of genetic divergence between nase individuals caught in rivers from the Danube drainage and invasive nase of Rhine origin sampled within the Rhône drainage, relative to the divergence observed among $C$. nasus populations sampled from different tributaries of the Danube (Gollmann et al. 1997). Little other phylogeographic and population genetic research has been carried out on this species across its range, especially in Switzerland and the Rhine drainage. Therefore the principal genetic units for conservation management within $C$. nasus remain largely unknown.

In this study we employ a novel conservation genomic strategy to survey neutral and adaptive genetic diversity across most extant (and one extinct) Swiss populations of the regionally imperilled $C$. nasus. A species for which there is little genomic information available. First we compared genotypic data from mtDNA, microsatellites and AFLPs to uncover the structuring of neutral genetic diversity among the Swiss nase populations across different time-scales. Second, age-structure data was combined with microsatellite and AFLP genotypic data to infer recruitment levels and potential inbreeding within each population. Finally, we employ a 'phenotype-down/genotype-up' approach to assay levels of local adaptation; $F_{\mathrm{ST}}$ outlier detection methods in conjunction with logistic regressions and morphological data were used to gauge relative levels of adaptive divergence among nase populations and to identify phenotypic traits underlying local adaptation. These data sets were then integrated and used to delineate distinct CUs within Swiss C. nasus and to inform future population management programmes.

\section{Methods}

Sampling and DNA extraction

The sampling scheme was designed to represent as much as possible all extant nase populations in Switzerland (Huber
Gysi 2009). In 2008, nase were caught in ten locations by electro-fishing (Table 1; Fig. 1, 2a). All fish were anesthetized with MS222 (Sandoz, Basel), measured, weighed and sexed in the field. When possible, three scales were removed for age determination. Standardized digital images were taken for geometric morphometric analyses. A small piece of anal fin tissue was preserved in absolute Ethanol for DNA extractions. Following the collection of field data, fish were allowed to recover from the anaesthetic and were released back into the wild at the sampling site. Additionally samples from a population in the Danube drainage (Na), samples from an invasive C. nasus population in the Rhône drainage (Doubs, Do) and historical samples from an extinct Swiss population (Sense, Se) were included in our analyses. Finally, an outgroup sample of Parachondrostoma toxostoma from the Rhône drainage were used in genetic analyses. Total DNA was extracted using a BioSprint 96 extraction robot (QIAGEN, Basel, Switzerland) with corresponding standard extraction method.

\section{Scalemetry and morphological measurements}

The age of individual fish from the six populations, for which scale samples were available ( $\mathrm{Bi}, \mathrm{Wi}, \mathrm{Mu}, \mathrm{Ro}, \mathrm{Li}$, $\mathrm{Ri}$ ), was estimated by counting the annular rings on scales using a binocular lens. Digital pictures were used for a landmark-based analysis of body shape variation. Twelve homologous landmarks, selected based on standard landmark descriptions (Bookstein 1991), were identified on $C$. nasus images (Fig. 3) and were set using the TPSDIG program (Rohlf 2006). Unless specified, all subsequent geometric morphometric (GM) analyses were carried out in MorphoJ 1.02d (Klingenberg 2011). Raw landmarks configurations were subjected to Generalized least-squared Procrustes super-imposition. Generated Procrustes coordinates were corrected for size-related allometry using pooled population-specific regressions against individual standard length. Size-corrected Procrustes coordinates were then used for further multivariate analyses. To investigate the contribution of age, sex and population of origin to overall shape variation, MANOVAs and discriminant function analyses (DFA) of these factors were carried out, with individual shape variation as a response. For DFA, all pairwise comparisons between groups within factors were tested: age [non-adult $(<4$ years) vs. adult $(\geq 4)$ ], sex (Male vs. Female), population of origin ( $\mathrm{Bi}, \mathrm{Wi}$, $\mathrm{Mu}, \mathrm{Li}, \mathrm{Su}, \mathrm{Ro}, \mathrm{Ri}$ ). For the MANOVAs, PCA of generated covariance matrices revealed five principal components (PCs) explaining $>5 \%$ of individual shape variation. As PC 1 was an artefact of fish body flexion during photography it was discarded. Individual PC scores along the remaining four PC axes were used as response variables, 


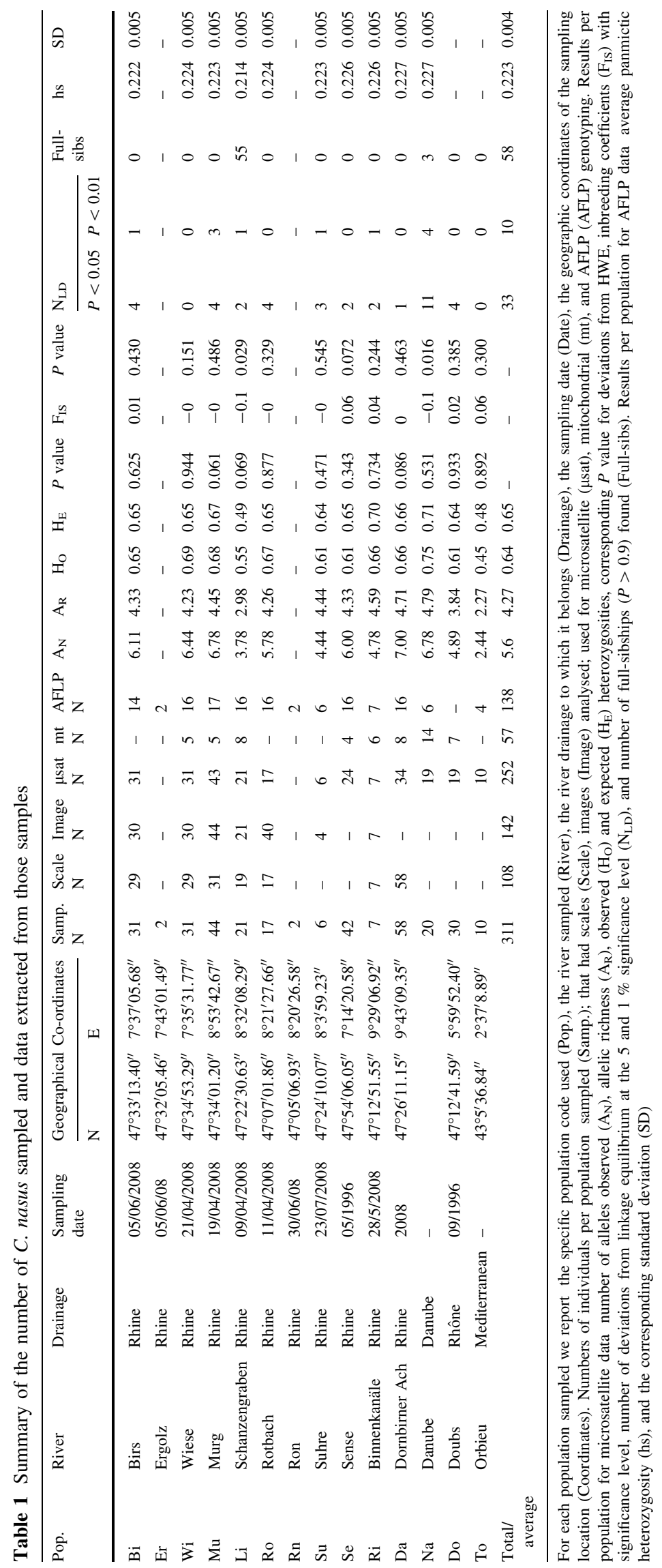




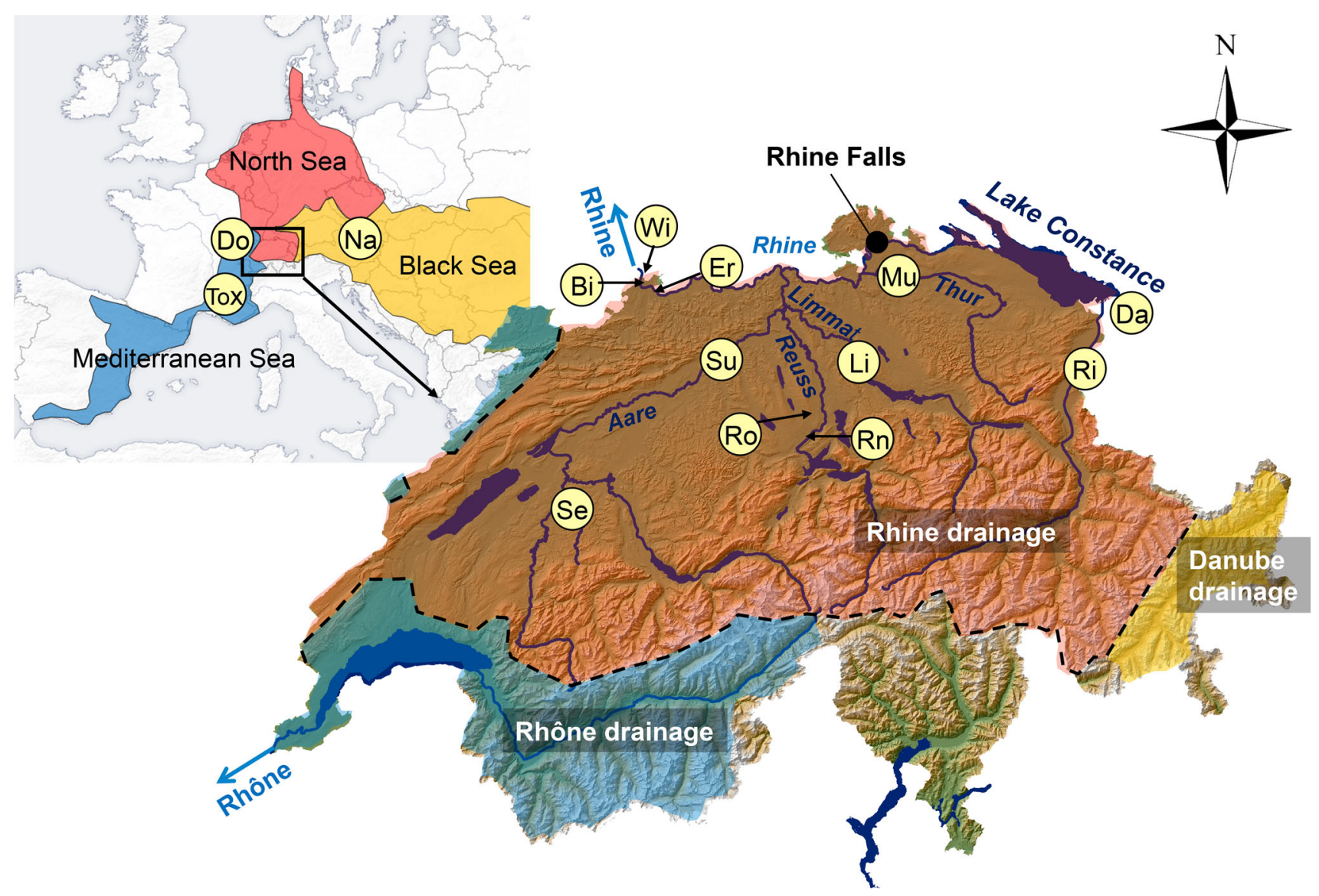

Fig. 1 Map ( ${ }^{\odot}$ Swisstopo) showing the nase sampling locations and the major hydrological/geographical features pertinent to this study. Coloured shading of map regions is representative of the major river

with individual age, sex and population membership as fixed factors. All MANOVAs were carried out in SPSS 18 (SPSS Inc., Chicago Illinois, USA). Following removal of fish less than four years old, CVA and Mahalanobis distances for the remaining 118 individuals from six populations (Bi, Wi, Li, Mu, Ri, Ro) were calculated to identify and quantify the major morphological differences between sampled populations.

\section{MtDNA phylogeographic analysis}

Portions of the mitochondrial genome covering 952 base pairs (bp) of the cytochrome $b$ and control regions were sequenced for 57 individuals selected from eight populations (Table 1), using primers and protocols described in Mäkinen and Merilä (2008). Sequences were resolved on an automated capillary loading DNA sequencer (CEQ 8000, Beckmann and Coulter, Fullerton, CA, USA) following manufacturer protocols and guidelines. Sequences were aligned using Sequencher v. 4.9 (Gene Codes Corporation) and verified by eye. The alignment was collapsed drainage basins found in the range of the Swiss nase: Rhine (red North Sea outflow), Danube (yellow Black Sea outflow) and Rhône (blue Mediterranean Sea outflow). Population codes as in Table 1

into the representative haplotypes with COLLAPSE v. 1.2 (http://darwin.uvigo.es/software/collapse.html), with preservation of individual haplotype frequencies. To investigate the demographic processes shaping genetic diversity in all sampled $C$. nasus populations, mismatch distribution analyses, Tajima's $D$, and $\mathrm{Fu}^{\prime}$ 's $F S$ neutrality tests were performed in ARLEQUIN 3.5 (Excoffier and Lischer 2010). These analyses were then repeated with the Danube population's haplotypes removed.

Microsatellite population genetic analysis

All specimens were genotyped at nine microsatellite markers (Mesquita et al. 2003; Vyskocilova et al. 2007; Muenzel et al. 2007). Because of overlapping allele size ranges of markers, two loci sets were generated for pre-PCR multiplexing (Set 1: SARN7G5, LSOU08 Cy5, SARN2F11B, LC290 Cy7, SARN7K4, LSOU21 Cy5.5; Set 2: LSOU05 Cy5, SARN7F8 Cy7, LC27 Cy5.5). Forward primers were labelled with fluorescent dyes. Multiplex PCR amplification was performed using a QIAGEN Multiplex PCR kit according to the 


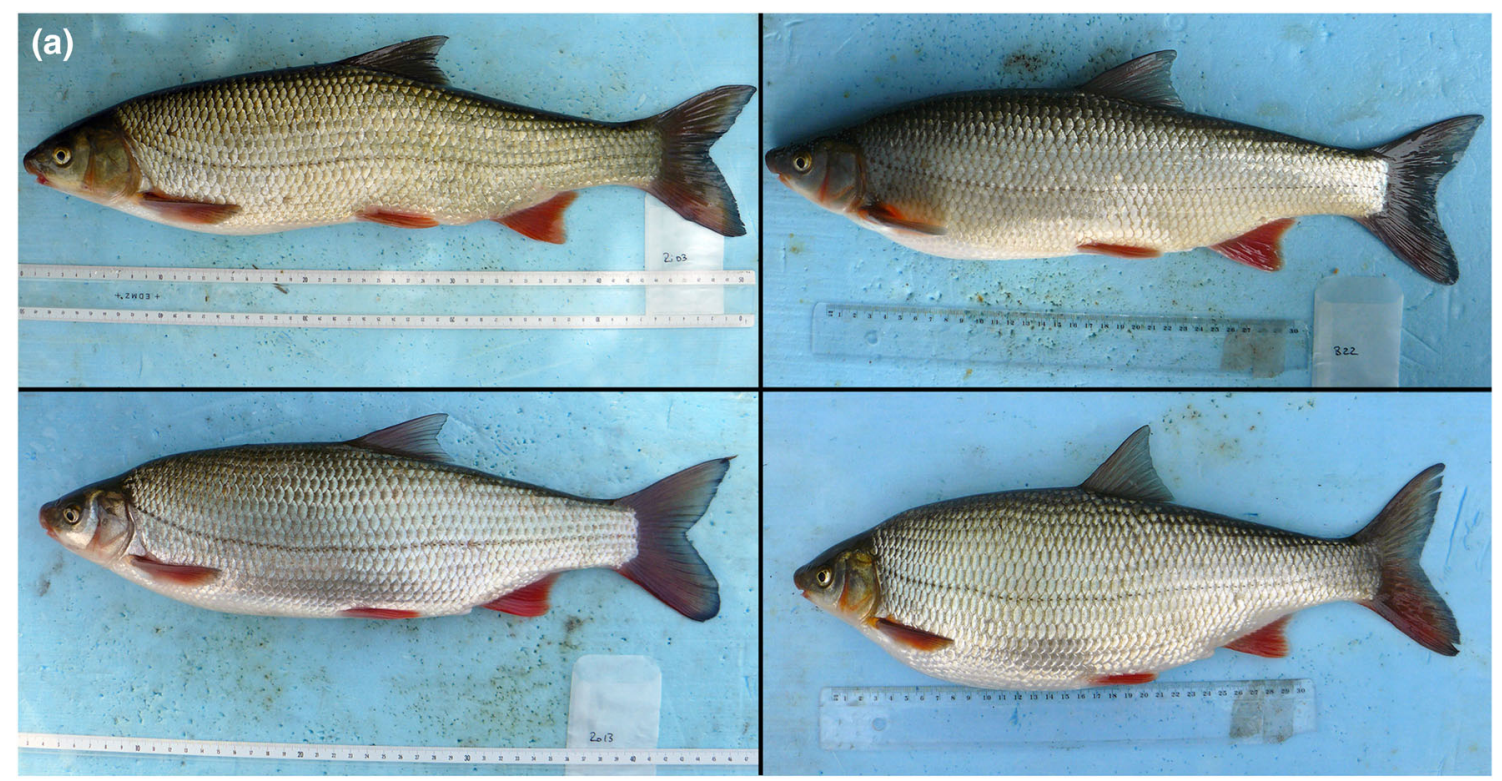

(b)
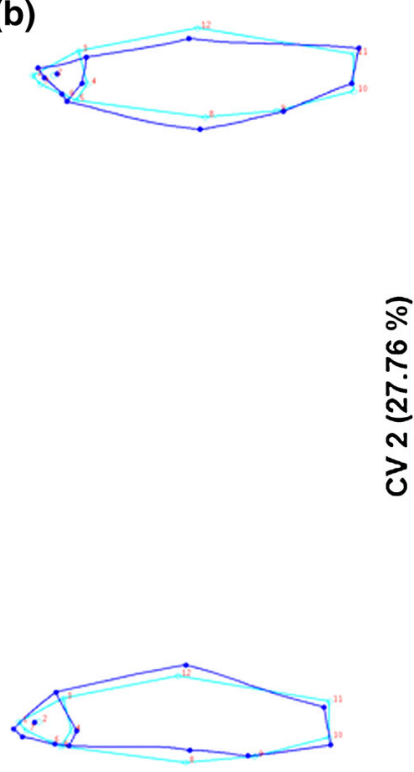

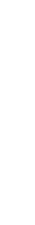

- Birs

- Wiese

- Murg

o Rotbach

- Schanzengraben

- Binnenkanäle
Fig. 2 Morphological diversity of Swiss nase. a Representative morphologies from different populations. From upper left clockwise: Binnenkanäle, Wiese, Schanzengraben, Rotbach. b Results of CVA of geometric morphometric data from Swiss populations. Dark blue

manufacturer's protocols. Fragments from each PCR product were resolved on a CEQ 8000 DNA sequencer. Microsatellites were scored using the provided fragment analysis software (Beckman Coulter, Fullerton, CA, USA). All allele designations were verified by eye. landmark configurations demonstrate the morphological changes along each CV relative to a standard configuration (light blue). Lower Rhine drainage populations are represented by filled circles, Lake Constance populations by filled squares

For each population expected $\left(H_{\mathrm{E}}\right)$ and observed $\left(H_{\mathrm{O}}\right)$ heterozygosities were calculated using ArLEQUiN. Deviations from Hardy Weinberg equilibrium (HWE) were tested with Fisher's exact tests (Guo and Thompson 1992). This was carried out for each locus and each population 


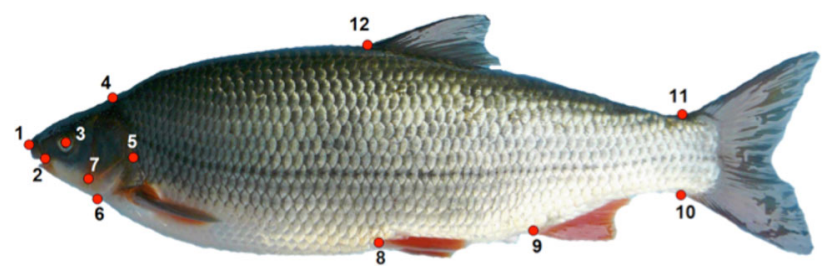

Fig. 3 Locations of the twelve homologous landmarks used for geometric morphometric analyses

using GenePoP 3.4 (Raymond and Rousset 1995), with 1,000,000 Markov chain steps and 5,000 dememorization steps. Allelic richness $\left(A_{\mathrm{R}}\right)$ was calculated in Fstat version 2.9.3 (Goudet 2001). Inbreeding coefficients $\left[F_{\text {IS }}\right.$ (Weir and Cockerham 1984)] were calculated across all loci for all populations and tested for significant deviations from zero using Fstat. $P$-values for deviations from HWE and for $F_{\text {IS }}$ were corrected for multiple comparisons using the sequential Bonferroni method (Rice 1989). Deviations from linkage equilibrium between all pairs of loci for each population were tested using ARLEQUIN. The presence of closely related individuals (family effects) was tested using COLONY v. 2.0.1.4 (Jones and Wang 2010). COLONY analyses were performed for each population using a medium run length, assuming polygamy in both sexes. Runs were performed both with and without the assumption of inbreeding. Full-sib relationships were considered significant if $P$ was greater than 0.9 (Junker et al. 2012). To test whether the medium length run was sufficient and that algorithms converged and yielded the same results, each analysis was repeated. The program BotTLENECK v. 1.2.02 (Cornuet and Luikart 1997) was used to detect whether sampled nase populations had experienced significant recent declines in effective population size. Expected heterozygosity calculated within each nase population was compared to an estimation of the heterozygosity expected in a population at equilibrium. A two-phase mutation model (TPM) was implemented in this analysis, assuming that $90 \%$ of the mutations followed a stepwise mutation model (SMM) and only $10 \%$ followed an infinite allele model. This mutation model was used as microsatellites are expected to mostly, but not fully, follow a SMM (Di Rienzo et al. 1994).

The global genetic differentiation (Global $F_{\mathrm{ST}}$ ) of populations in different grouping scenarios [(1) Rhine drainages below Lake Constance (hereafter lower Rhine) vs. Danube/ Rhine drainages draining into Lake Constance (hereafter Lake Constance); (2) lower Rhine/Lake Constance vs. Danube; (3) lower Rhine vs. Lake Constance vs. Danube] were calculated in hierarchical analyses of molecular variance (AMOVAs) using ARLEQUin. The number of private alleles found within each geographical population grouping and the relative number of private alleles, corrected for the number of individuals genotyped for each group, were calculated from raw allele frequencies data. A Bayesian population inference algorithm, implemented in STRUCTURE 2.3.3 (Pritchard et al. 2000), was used to infer the number of genetically distinct clusters represented by the sampled nase populations. STRUCTURE run parameters were: 50,000 step burn in; 500,000 MCMC steps; ten iterations for each K, from one to thirteen; admixture and LOCPRIOR models; correlated allele frequencies. For the LOCPRIOR model the sampling sites for each population were encoded as discrete locations, with no information on the geographic distance between sites. To identify potential hidden population subgroups, additional STRUCTURE analyses were run separately on the populations within each of the genetic clusters identified by the initial Structure run. To visualize population differentiation and similarity, a PCA based on population allele frequencies was performed in PCAGEN (Goudet 1999). METAPOP version 2.0 (Pérez-Figueroa et al. 2009) was used to assess the relative contribution of each population to overall nase genetic diversity. This analysis was first run using all sampled nase populations and then on a subset comprising only populations from the lower Rhine genetic cluster.

\section{AFLP population genetic analysis}

We used a modified version of the original protocol introduced by Vos et al. (1995), with the restriction and ligation steps combined in a single reaction mix. In the selective amplification stage, selective PCRs were performed with nine different EcoRI/MseI primer-pairs. The nine primer-pairs were analysed in three different multiplex-sets (Set 1: CTT ACA, CTT AAG, CTT AGC; Set 2: CTC ACA, CTC AAG, CTC AGC; Set 3: CTA ATT, CTA AGG, CTA ATC). Fragment separation was carried out using a CEQ 8000 DNA sequencer. The resulting AFLP traces were then analysed with GENEMARKER v 1.85 (SoftGenetics, LLC, State College, PA, USA). Only fragments sized between 60 and 400 base pairs in length were scored. Scoring of loci was carried out using an automatic scoring algorithm, followed by manual inspection. To verify levels of reproducibility of the AFLP process, 36 to 41 individuals were repeated per primer-pair ( 25 to $30 \%$ of total). The average \% level of mismatch across all independent replicate pairs was then reported for each primer pair (Bonin et al. 2004).

Both individual-based and population-based NJ phylogenetic trees were reconstructed from AFLP loci using the program NeIGHBoR in the software package PHYLIP 3.67 (Felsenstein 1989). Individual pair-wise genetic distances were calculated from 553 AFLP loci using RESTDIST in PHYLIP with 1,000 bootstrap replicates. Four P. toxostoma 
individuals were used as an outgroup. Nei's genetic distances between $C$. nasus populations were calculated in AFLP-SURV 1.0 (Vekemans 2002) and used to construct population-based trees. $\mathrm{Er}$ and $\mathrm{Rn}$ individuals were excluded from the population-based trees due to the limited number of fish sampled. To investigate the number of genetic clusters within sampled $C$. nasus, individual-based Bayesian population assignments were carried out on all genotyped nase individuals in STRUCtURE. Runs consisted of a 100,000 burn-in, followed by 500,000 MCMC repetitions. All other parameters were the same as those used in the microsatellite Structure analysis. Additional StrucTURE analyses were again run separately within each of the genetic clusters identified. To investigate the partitioning of genetic variation among and within the major genetic clusters identified by Structure, Arlequin was used to carry out an AMOVA on the AFLP data, with 10,000 permutations. The average panmictic heterozygosity within each population $(h s)$ was calculated for AFLP data in HicKORY 1.1 (Holsinger and Lewis 2007), with a burn-in of 50,000 , followed by 250,000 repetitions.

Detecting loci and phenotypic traits linked to local adaptation

To identify the locus-specific signal of divergent natural selection and therefore of local adaptation between the different $C$. nasus populations, two different $F_{\mathrm{ST}}$ outlier detection approaches were used to screen individual AFLP genotypes: DFDIST D (Beaumont and Balding 2004) and BAYESCAN v. 2.1 (Foll and Gaggiotti 2008). As hierarchical genetic structuring of populations can lead to an increase in false positive candidate loci being found during $F_{\mathrm{ST}}$ outlier analysis (Thornton and Jensen 2007; Excoffier et al. 2009), nase populations were divided into data sets comprising the major genetic clusters identified, each cluster being analysed separately. The first data set consisted of seven nase populations from catchments below Lake Constance ( $\mathrm{Li}$, $\mathrm{Wi}, \mathrm{Mu}, \mathrm{Bi}, \mathrm{Su}, \mathrm{Ro}, \mathrm{Se})$. The second consisted of two populations from the rivers draining into Lake Constance (Ri, Da). Due to limited sampling, Danubian (Na) individuals were not used in outlier detection analysis. For each genetic cluster data set, outlier detection analysis was first carried out on all populations simultaneously (global analyses), followed by all within-cluster pairwise population comparisons (pairwise analyses) (Bonin et al. 2006). To screen for the genomic signal of adaptive divergence between the two Swiss nase population groupings, yet avoid biases associated with the hierarchical structuring of genetic variation, pairwise population $F_{\mathrm{ST}}$ outlier comparisons were carried out between each of the populations from the lower Rhine ( $\mathrm{Li}, \mathrm{Wi}, \mathrm{Mu}, \mathrm{Bi}, \mathrm{Su}, \mathrm{Ro}, \mathrm{Se})$ and each of those from the Lake Constance cluster (Ri, Da).
DFDIST estimates the probability of a locus to be under selection according to its observed $F_{\mathrm{ST}}$ and $H_{\mathrm{E}}$, compared to simulated neutral distributions, computed for an average "neutral" $F_{\mathrm{ST}}$ observed in the empirical data set (Beaumont and Balding 2004). The mean $F_{\mathrm{ST}}$ used for simulating a null distribution of $F_{\mathrm{ST}}$ values for loci evolving under neutrality, was estimated using a trimmed mean of 0.3 . The null distribution was generated using 50,000 loci. In the empirical AFLP data set the maximum allele frequency was set to 0.98 . AFLP loci with pairwise $F_{\mathrm{ST}}$ values above the 0.95 quantile of the null distribution were considered candidate loci for being under divergent natural selection, following multitest correction at a false discovery rate (FDR) of 0.05. Multitest correction was carried out using the FDR test of Benjamini and Hochberg (1995) and the SGoF test of Caravajal-Rodriguez et al. (2009), as implemented in $\mathrm{SGoF}+$.

In contrast, BAYESCAN uses a hierarchical Bayesian approach, allowing a direct estimation of the probability of non-neutrality for each locus, rather than a userdetermined cut-off value for the rate of false positives (Foll and Gaggiotti 2008). Model parameter estimation was automatically tuned during the pilot runs (50 runs, length 5,000). A 50,000 iteration burn-in was found to be sufficient for convergence between MCMC chains. Sample sizes of 50,000, a thinning ratio of 10 and prior odds for the neutral model of 10 , were found to give stable results in repeated analysis runs and were used as parameters for all subsequent analyses. Only loci below a FDR of 0.05 were considered as divergent selection candidate loci.

Logistic regressions were used to calculate the significance of association between pair-wise models of AFLP allele scores (presence/absence) and phenotypic trait values, in comparison to models utilizing a constant (Jump et al. 2006; Joost et al. 2007). Univariate logistic regressions were carried out in the program MATSAM 2BETA (Joost et al. 2007). The data set for MATSAM analysis comprised 62 adult individuals belonging to five populations in the lower Rhine genetic cluster (Wi, Bi, $\mathrm{Li}, \mathrm{Mu}$, Ro). For Lake Constance populations, comparable phenotypic/genotypic data were only available for six individuals from the Ri population. Due to the small sample size and potential confounding effects of hierarchical structuring of genetic variation among major population groupings, Lake Constance individuals were removed from this analysis. Individual AFLP genotypes were regressed against each of the corresponding individual PC 2-5 scores resulting from the GM analyses of shape. Individual trait values used in logistic regressions can be found in Online Resource 1. The significance of the association between the frequency of alleles at a locus and the value of each trait parameter was assessed using two test statistics: $G$ and the more stringent 
Wald test, with the FDR for testing multiple models set to 0.05 in $\mathrm{SGoF}+$, correcting for the number of AFLP loci used in each regression analysis.

\section{Results}

Age structure

Age structure of nase varied among sampling locations, with only the Da population having the full range of age classes $(0+$ to $15+)$ (Online Resource 2$)$. The populations with the most restricted number of age classes sampled were $\mathrm{Li}(3+$ to $7+)$ and $\mathrm{Ri}(3+, 12+, 13+, 15+)$, although the latter comprised a sample of only six fish. The remaining populations had more even, wider distributions of age classes.

\section{Morphological analysis}

DFA and MANOVA found no significant effect of sex on overall body shape variation among individuals, with no interactive effect with population of origin, so this factor was ignored in further GM analyses. DFAs using age showed significant differences between age classes and assignment of individuals to age groupings $\left(t^{2}=47.52\right.$, $P=0.01)$. MANOVAs using age as a factor were narrowly non-significant (Wilks' Lambda: $F=1.436$, $P=0.058$ ), including the interaction term with population of origin $(F=1.369, P=0.11)$. Overall MANOVA results showed population of origin to be the factor explaining the largest amount of shape variation ( $F=3.273, P<0.001)$. To remove potential biases due to differences in age structure among population samples, individuals less than four years old were removed from further GM analyses. Following CVA and permutation tests, Mahalanobis distances between populations were found to be significant in all pairwise comparisons (2.05 to 6.25, $P \leq 0.001)$. CV 1 (45.9\%) separated populations with relatively small heads and deeper bodies (more negative scores; Li population), from those with larger heads and more streamlined bodies (most other nase populations) (Fig. 2a, b). CV 2 (27.8 \%) distinguished populations with more down-turned snouts (more negative scores; Ro and $\mathrm{Ri}$ ) from those with up-turned snouts $(\mathrm{Bi}, \mathrm{Mu}, \mathrm{Wi}$ and $\mathrm{Li}$ ). Of the four non-artefactual PCs found during PCA, individuals with higher PC 2 scores $(17.0 \%)$ were deeperbodied with smaller, more down-turned heads. Individuals with higher PC $3(10.1 \%)$ scores had larger heads and more streamlined bodies. Higher PC $4(8.6 \%)$ scores indicated a longer head and higher PC $5(6.5 \%)$ scores were associated with more narrow, down-turned heads (Online Resource 3).
MtDNA sequencing and phylogeographic analysis

Only five mtDNA haplotypes were recovered from the eight populations sampled (GenBank accession numbers: KJ603523 to KJ603527), separated by a maximum of six mutational steps (Fig. 4). Three of these haplotypes (N1, N3, N5) were unique to Danubian individuals (Na): making this population the most diverse. Haplotype N4 was found only in individuals sampled from Rhine catchment drainages below Lake Constance ( $\mathrm{Li}, \mathrm{Mu}, \mathrm{Wi}, \mathrm{Se}$ ) and the introduced Do population. Haplotype N2 was present in populations from rivers draining into Lake Constance (Ri, Da), certain populations from rivers below Lake Constance $(\mathrm{Wi}, \mathrm{Mu}$ ), and the invasive Do population (Online Resource 4). Mismatch analyses revealed near-significant support for models of sudden population expansion in both the total population and non-Danubian mtDNA data sets (sum of squared deviations, $P=0.052$ and $P=0.089$ respectively) (Online Resource 5). In contrast, the values obtained for Harpending's raggedness index were significant, rejecting a model of demographic expansion $(r=0.29, P=0.013$ and $r=0.75, P=0.012)$. For the entire nase mtDNA data set, Tajima's $D$ and Fu's $F S$ statistics were positive and non-significant $(1.47, P=0.91$ and $1.79, P=0.84$ respectively). When Danubian haplotypes were removed, these statistics became more strongly positive (2.48, $P=1.0$ and $5.35, P=0.98$ ). The results of both Tajima's $D$ and Fu's $F S$ tests indicate a general lack of low frequency haplotype variants among the individuals sampled, potentially indicating recent population declines.

\section{Microsatellites population genetic analysis}

Within populations, no significant deviations from HWE and no significant $F_{\text {IS }}$ values were observed at any of the microsatellite loci or in any of the populations after sequential Bonferroni correction (Table 1). The $F_{\text {IS }}$ of two populations, $\mathrm{Li}$ and $\mathrm{Na}$, were significant before multitest correction. The tests for deviations from linkage equilibrium were significant in 29 of 432 comparisons at $P<0.05$ $(6.71 \%)$ and in seven of 432 at $P<0.01(1.62 \%)$. Only one population $(\mathrm{Na})$ showed a slightly increased frequency of LD. Within populations, the mean number of alleles per locus ranged from 2.44 to $6.78, H_{\mathrm{E}}$ ranged from 0.48 to 0.71 , and $A_{\mathrm{R}}$ ranged from 2.27 to 4.79 (Table 1). Within the Rhine drainage, genetic diversity was particularly low in the $\mathrm{Li}$ population $\left(A_{\mathrm{R}}=2.98\right)$. The BotTLENECK analysis showed that the expected heterozygosity was in all lower Rhine populations significantly larger than that expected under an assumption of population equilibrium, supporting strong and recent population declines in most populations. Although expected heterozygosity was higher than at population equilibrium in both Lake Constance populations, this 


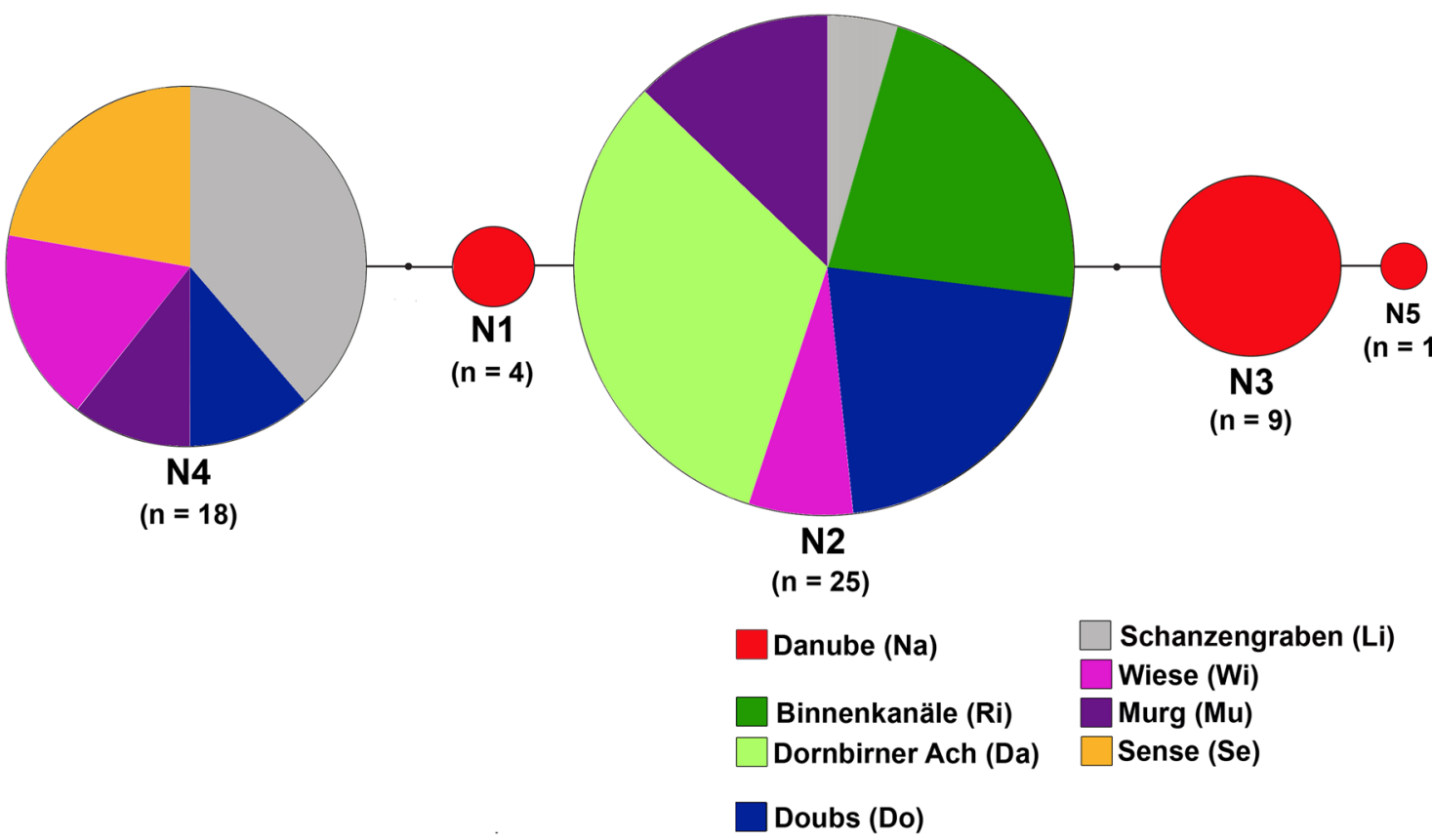

Fig. 4 Unrooted mtDNA haplotype network of sampled C. nasus individuals. Circles correspond to unique haplotypes: their size representing haplotypic frequency among individuals. Colours refer

was found to be non-significant (Online Resource 6). Pairwise estimation of sibship within populations found high likelihoods for the existence of full-sibling relationships only among individuals from the $\mathrm{Na}$ (three comparisons) and the Li populations (55). Assuming inbreeding during the analysis run had no effect on the number of significant sibship comparisons found. The high numbers of full-sibships in the Li population suggests that the individuals caught in this river stem from very few parents (eleven out of the 21 individuals were assigned to one family). For the Na population, the increased $F_{\text {IS }}$, the slightly increased LD and the three significant full-sib comparisons may be due to this sample consisting solely of captive-bred F1 individuals, resulting from the crossing of a small number of wild-caught nase (three males and five females).

Among Rhine drainage populations, we found considerable variation in genetic differentiation among populations, with pairwise $F_{\mathrm{ST}}$ values ranging from 0 to 0.213 (Table 2). Three patterns could be observed within the Rhine drainage: First, the Li population was genetically very distinct from all other populations $\left(F_{\mathrm{ST}}\right.$ ranged from 0.107 to 0.213 ). Second, the two populations sampled from rivers flowing into Lake Constance ( $\mathrm{Ri}, \mathrm{Da})$ were very distinct from other Rhine drainage populations $\left(F_{\mathrm{ST}}\right.$ ranged from 0.079 to 0.213$)$, but not from each other $\left(F_{\mathrm{ST}}=0\right)$. Third, among the remaining Rhine populations downstream of Lake Constance (Bi, Wi, Mu, Ro, Su, Se), even though they may be geographically distant from one to individual sampling location of origin. Connections in black between haplotypes represent mutational steps. Black dots represent missing haplotypes

another, no significant neutral genetic differentiation could be observed (Table 2). The invasive nase population (Do), sampled from the Rhône drainage, showed generally low but significant levels of genetic differentiation from most lower Rhine populations $\left(F_{\mathrm{ST}}\right.$ ranged from 0.021 to 0.047 ; $\mathrm{Li}$ pairwise $F_{\mathrm{ST}}=0.175$ ). Levels of pairwise genetic divergence between lower Rhine populations or Lake Constance populations with the Danubian population were generally similar ( $F_{\mathrm{ST}}$ ranged from 0.09 to 0.243 and 0.082 to 0.095 respectively). $F_{\mathrm{ST}}$ values between all $C$. nasus populations and the outgroup $P$. toxostoma were very high $\left(F_{\mathrm{ST}}\right.$ ranged from 0.343 to 0.445 ; Table 2$)$. A K of two for all nase populations was found to have by far the highest $\Delta K$ (965) in STRUCture. The first genetic cluster grouped the Lake Constance populations together with Danubian nase (Fig. 5a). The second grouped all lower Rhine populations, including the $\mathrm{Li}$ population, and the invasive Do population from the Rhône drainage. STRUCTURE analysis solely of populations within the lower Rhine cluster, revealed a $\mathrm{K}$ of three to be the most likely [mean Ln $\mathrm{P}(\mathrm{K})(-4,529)]$. The distinct groupings within the lower Rhine cluster comprised: (i) the $\mathrm{Li}$ population, (ii) the invasive Do population, (iii) all remaining lower Rhine populations. No additional population subgroups were identified by STRUCTURE within the Lake Constance cluster. PCA depicted the distinctiveness of $\mathrm{Li}$ and confirmed the closer genetic relationship between Lake Constance and Danube populations (Fig. 5b, c). A single individual 
sampled in the Lake Constance tributary Dornbirner Ach (Da) showed a microsatellite genotype representative of populations from the lower Rhine cluster. Following the re-extraction of its DNA and repeat microsatellite genotyping, this individual's genotype was confirmed and was therefore removed from all population-based analyses. AMOVA results showed that separating nase populations into three groups based on their broad geographic origin (lower Rhine, Lake Constance, Danube), explained the most genetic variance $\left(F_{\mathrm{CT}}=0.083, P<0.01\right.$; Online Resource 7). The private allele analysis showed that the lower Rhine group had eleven private alleles, the Lake Constance group had five and the Danubian population had nine. The lower Rhine and Lake Constance population groupings shared more alleles to the exclusion of the Danube population (fourteen), than either alone did with the Danubian population (eight and seven respectively). Adjusted for sample size, the frequency of private alleles in the Danube was highest $(0.45)$ followed by the Lake Constance (0.077) and the lower Rhine (0.045). In the METAPOP analyses, removal of the Da and Na populations had the greatest negative effect on overall genetic diversity (1.6 and $1 \%$ ) and allelic richness (2.2 and $4.6 \%$ ), due to positive contributions from both the within population and between population components of the overall measure of genetic diversity (Online Resource 8). Removal of most individual lower Rhine populations actually lead to an increase in overall genetic diversity $(-0.1$ to $-0.6 \%)$ and allelic richness $(-0.16$ to $-0.55 \%)$, largely due to the low levels of genetic distinctiveness among these populations. Carrying out the METAPOP analyses using only lower Rhine populations, the Mu population made the largest contribution to both overall genetic diversity $(0.7 \%)$ and allelic richness (1.05). In this analysis, the Li population was found to contribute the lowest amounts of unique within-population genetic diversity ( -2.6 vs. -0.16 to $1.66 \%$ ). However, the overall contribution of the Li population to total genetic diversity $(-0.1 \%)$ was balanced by the large genetic distance between this population and other lower Rhine populations $(2.5 \%)$. Removal of the $\mathrm{Li}$ population had the greatest effect on overall allelic richness in the lower Rhine nase populations: $-2.4 \%$ as compared to -0.36 to $1.05 \%$ for the other populations.

\section{AFLP population genetic analysis}

For the nine primer pairs scored, 138 C. nasus individuals from twelve sampling locations and four $P$. toxostoma individuals were genotyped for a combined total of 553 AFLP loci (Table 1). Levels of reproducibility were high, varying from 96.9 to $98.4 \%$ among primer pairs. Identification codes, total number of loci, number of polymorphic loci and average reproducibility for each AFLP primer pair

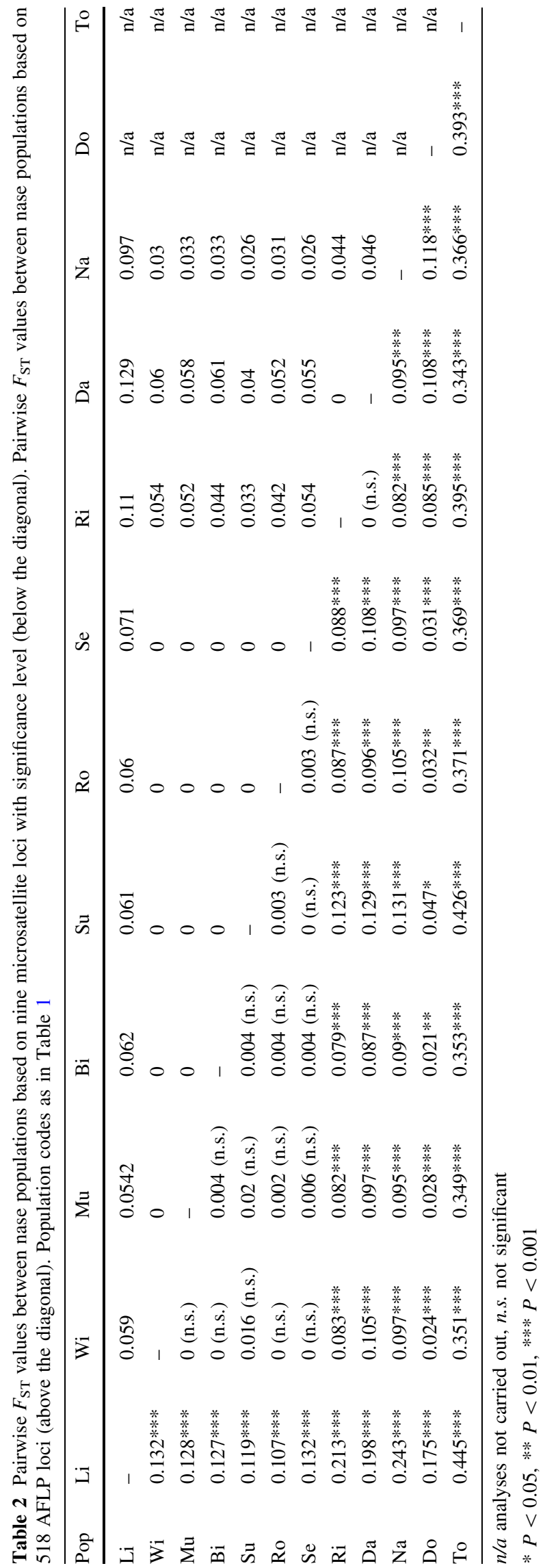


(a) 9 Microsat. Loci, $\mathrm{K}=2$

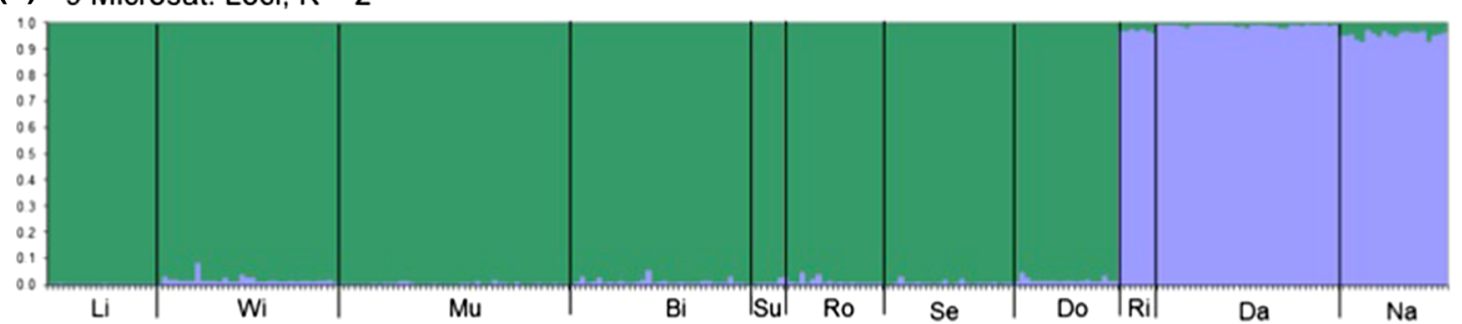

(b)

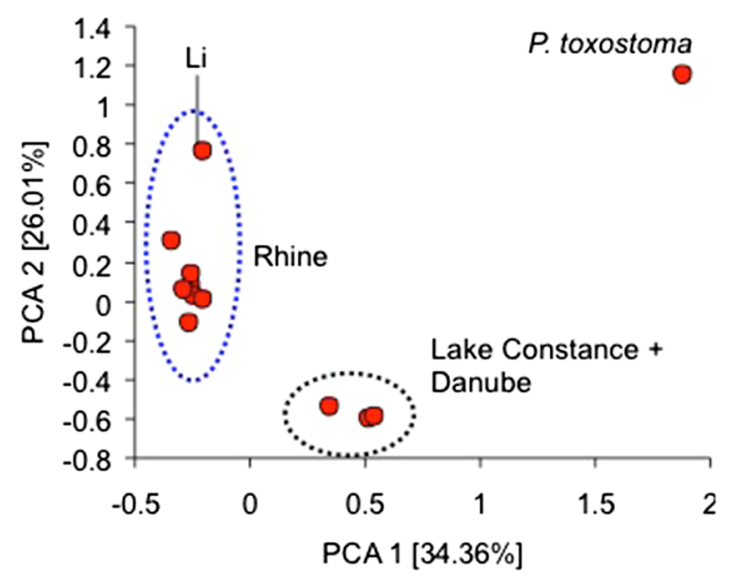

Fig. 5 Population structure of nase in Switzerland as inferred from microsatellite data. a Individual population assignments estimated by STRUCTURE for $\mathrm{K} 2$ 2. PCA based on population allele frequencies:

can be found in Online Resource 9. Individual-based AFLP phylogenetic trees strongly supported the monophyly (100\% bootstrap support) of all genotyped C. nasus individuals (Online Resource 10). Within the $C$. nasus clade, individual genotypes were generally clustered into three major groups that corresponded to three geographically distinct areas: Danube, Rhine drainages below Lake Constance and Rhine drainages above Lake Constance. However re-sampling support for these groups was low $(<50 \%)$. Within these geographic groupings, there was little obvious structuring of individuals by sampling location, with the exception of the Li population: 15 out of the 16 individuals genotyped formed a weakly supported group (25\%). Population-based AFLP trees revealed similar, but better supported, topologies (Online Resource 11). Three major clades were revealed that corresponded to the distinct geographic units found in individual-based trees: lower Rhine (Bi, Wi, $\mathrm{Li}, \mathrm{Mu}, \mathrm{Ro}, \mathrm{Su}, \mathrm{Se}$ populations) (64\% bootstrap support), Lake Constance (Ri, Da) (100 \%) and Danube (Na) (97\%).

Following visualization of the AFLP STRUCTURE results for all nase populations, a $\mathrm{K}$ of three was found to have the highest mean $\operatorname{Ln} \mathrm{P}(\mathrm{K})(-30,931)$ and the highest $\Delta \mathrm{K}(167)$. The first genetic cluster comprised all individuals sampled from River Rhine tributaries below Lake Constance. The second cluster comprised all individuals sampled from (c)

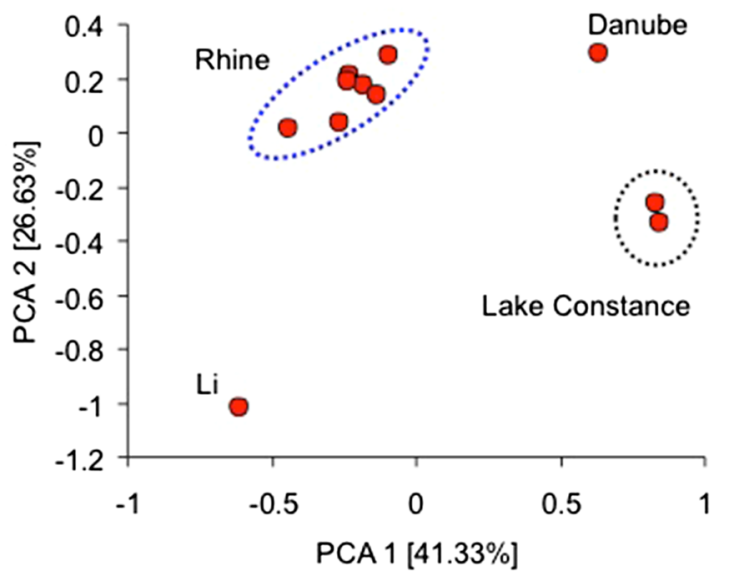

b with all populations included; c P.toxostoma excluded. Population codes as in Table 1

rivers flowing into Lake Constance. The final genetic cluster comprised those individuals of Danubian origin (Online Resource 11). Additional Structure analyses revealed no hierarchical substructuring of genetic variation within each identified cluster. AMOVA of the hierarchical structuring of AFLP genetic variation in Swiss $C$. nasus revealed that most variation, other than among individuals $(86.10 \%, P<0.001)$, was sequestered among the three major geographic population clusters: lower Rhine, Lake Constance and Danube $(10.64 \%, P=0.001)$. Only $3.26 \%(P<0.001)$ of variation was structured among populations within these genetic clusters. Patterns of pairwise $F_{\mathrm{ST}}$ values between populations using AFLP loci were lower, but largely congruent with those involving microsatellite loci (Table 2). Among populations in the lower Rhine grouping (excluding population $\mathrm{Li}$ ), and among those of the Lake Constance drainages, $F_{\mathrm{ST}}$ values were not significantly different from zero. Pairwise comparisons of genetic divergence between populations from the lower Rhine and Lake Constance clusters revealed generally higher $F_{\mathrm{ST}}$ values (0.033 to 0.061) than comparisons involving populations from either Swiss cluster and the Danubian population ( 0.026 to 0.046 ). The highest $F_{\text {ST }}$ values were found in pairwise comparisons involving the $\mathrm{Li}$ population (0.054 to 0.129). Following analysis in 
Hickory, the full model was found to be the most appropriate $(\mathrm{DIC}=11,583.3)$. From this model, average panmictic heterozygosity ( $h s$ ) was found to be lowest in the $\mathrm{Li}$ population (0.214) compared to other sampled $C$. nasus populations [0.221 to 0.227 (Mean $=0.223)$; Table 1].

Detecting loci and phenotypic traits linked to local adaptation

For the Li population, eight individuals, out of the 16 AFLP genotyped, were found to be full siblings following the COLONY analysis. Seven of these individuals were subsequently excluded from the $F_{\mathrm{ST}}$ outlier detection data sets to reduce the potential for biases to the observed distribution of $F_{\text {ST }}$ values in comparisons involving this population. For the global comparison including all lower Rhine populations $(\mathrm{Li}$, $\mathrm{Wi}, \mathrm{Mu}, \mathrm{Bi}, \mathrm{Su}, \mathrm{Ro}, \mathrm{Se}$ ), for both outlier detection methods, the strongest candidate outlier locus was TCTA-318. However this locus was found to be significant only in the DFDIST analyses, following the $\mathrm{SGoF}$ multitest correction (DFDIST $F_{\mathrm{ST}}=0.244, \quad P=0.008, q=0.045 ;$ BAYESCAN $F_{\mathrm{ST}}=$ $0.043, q=0.762)$. The presence allele for this locus had a lower prevalence in the Li population $(33.33 \%)$ than in other lower Rhine populations ( 81.25 to $100 \%$ ). The results of the DFDIST and BAYESCAN pairwise lower Rhine population comparisons found no significant selection candidate loci. For the global analysis of Lake Constance populations ( $\mathrm{Ri}, \mathrm{Da}$ ), neither the DFDIST nor BAYESCAN analyses identified any significant $F_{\mathrm{ST}}$ outlier loci. For each outlier detection method, the closest locus to candidate outlier status was GCTC-241 (DFdist $F_{\mathrm{ST}}=0.384, P=0.004, q=1$; BAYESCAN $F_{\mathrm{ST}}=$ $0.038, q=0.82$ ). The presence allele at this locus was absent in the Ri population compared to $69 \%$ prevalence in the Da population. Pairwise comparisons between the lower Rhine and Lake Constance population groupings, revealed no significant $F_{\mathrm{ST}}$ outlier loci following multitest correction. The strongest candidate for outlier status was locus GGTA-257, which had a higher prevalence of the presence allele in the Lake Constance populations (71.43 to $81.25 \%$ ), compared to all lower Rhine populations (0 to $12.5 \%$ ) (DFDIST $F_{\mathrm{ST}}=$ $0.2870 .534, P=0.0030 .093, q=0.6761$; BAYESCAN $\left.F_{\mathrm{ST}}=0.0880 .21, q=0.5470 .9\right)$. Logistic regressions revealed no significant association between allelic variation at specific AFLP loci and individual scores along the major axes of body shape variation identified by geometric morphometrics.

\section{Discussion}

In this study, we used a multidisciplinary approach combining genetic, phenotypic and demographic data to investigate the geographic structuring of phenotypic and genetic (both neutral and adaptive) diversity across populations of endangered nase (C. nasus) in Switzerland. We have integrated these data sets to identify the principal CUs for the nase in its Swiss range and to better inform future conservation management strategies for the constituent nase populations within each CU. Based on neutral markers, we found two strongly differentiated, previously unknown, major population groupings within Swiss nase. The first major population grouping comprised those populations found in rivers flowing into Lake Constance and the second comprised all populations sampled from tributaries of the Rhine below Lake Constance. Amongst the constituent populations of the principal genetic clusters there were generally weak and non-significant levels of neutral genetic differentiation. The one exception to this pattern is the Schanzengraben (Li) population, which showed strong genetic divergence to all others within the lower Rhine cluster. A genomic screen for adaptive genetic variation among populations, both within and between the principal genetic clusters identified in this study, revealed only one significant selection candidate locus: TCTA-318, found in outlier comparisons involving the Li population. Phenotypic evidence for local adaptation came in the form of significant body shape differentiation among different nase populations. Nearly all sampled nase populations, at least within the lower Rhine genetic cluster, showed significant genetic trends supporting recent, rapid, negative population growth, confirming demographic observations as to the imperilled status of Swiss $C$. nasus. We suggest that the two geographically distinct genetic clusters identified in this study: (i) all Lake Constance and (ii) all lower Rhine populations, should be treated as distinct CUs. Overall, the approach employed in this study has proven to be useful in surveying the demographic processes underlying nase diversity in the alpine range. The methodological framework outlined in this study provides the necessary baseline knowledge for informed conservation management and is thus a important stepping-stone to enable more focused, hypothesis-driven studies in the future, which would allow a deeper insight into the evolutionary mechanisms that drive the observed morphological differentiation between populations, and to measure the full extent of local adaptation in this study system.

\section{Population structure and demography}

The two nuclear marker systems used in this study (AFLPs and microsatellites) showed remarkable consistency in the relative levels of population differentiation and measures of population genetic diversity found. Both marker types clearly showed the existence of two genetically distinct major populations clusters within Switzerland: one consisted of nase sampled from rivers draining into Lake 
Constance, the other centred on Rhine drainages downstream from Lake Constance. Physically isolating these two genetic clusters is the Rhine Falls, which has prevented the colonization of the Upper Rhine even by mobile fish species such as the Atlantic salmon (Salmo salar). Despite this barrier, unidirectional gene flow downstream from Lake Constance may still be possible and the sharing of mtDNA haplotype N2 (see below) and microsatellite alleles, perhaps indicates that this has happened to a limited degree. The third major population grouping revealed in this study consisted of the samples from the River Enns, in the Upper Danube. Similar levels of genetic differentiation among major geographical population groupings suggest near-simultaneous divergence between these clusters with limited subsequent gene flow since this time point. The discovery of private microsatellite alleles increases support for the evolutionary distinctness of each of these population groupings and at the same time reduces the likelihood that any of these genetic clusters were of recent hybridogenic origin, especially the geographically intermediate Lake Constance cluster. One note of caution, our Danubian nase sample was limited to the F1 captive-reared progeny of five males and three females taken from a single spawning site; therefore more work is needed to confirm that our sample is representative of Danubian nase in general, and to better understand the genetic structuring and demographic history of nase populations in this drainage.

Within each of the lower Rhine and Lake Constance genetic clusters, the constituent populations were more closely related to each other than to either those from the other cluster or the Danubian nase. Given that Lake Constance and the surrounding Swiss lowlands were glaciated until 12,000 to 15,000 years ago and the moderate level of genetic differentiation between clusters $\left[F_{\mathrm{ST}}=0.079\right.$ to 0.129 (microsatellites), 0.033 to 0.061 (AFLPs) (excluding comparisons with the Schanzengraben population)], this suggests that these clusters have diverged, in situ, since the end of the last ice age. The very low, non-significant levels of genetic differentiation among the constituent sampling locations within each of these units (except Schanzengraben: see below) also suggest high levels of gene flow, little population structure and dispersal across quite large geographic distances (e.g. more than $100 \mathrm{~km}$ between Sense and Wiese). It is predicted that declining, isolated populations should show increased genetic differentiation among populations due to genetic drift, and reduced genetic diversity within populations. However, even though nearly all populations show a genetic signal of having passed through a population bottleneck, they still show non-significant levels of genetic differentiation, even in comparisons with the now extinct population in the Sense River. Indeed, the Sense River population, had very similar levels of genetic diversity and heterozygosity to extant populations, even though these samples were taken only a decade prior to extirpation. This data strongly supports the theory that the main factor driving extinction in nase populations is not inbreeding in diminished populations, but instead that a loss of riverine habitat or connectivity between habitats, required by different life history stages, prevents successful natural recruitment, whilst adults may still thrive (Kirchhofer 1996; Huber and Kirchhofer 2001).

Although the Schanzengraben population showed consistently high levels of genetic differentiation from other Swiss nase populations $\left[F_{\mathrm{ST}}=0.107\right.$ to 0.213 (microsatellites), 0.054 to 0.129 (AFLPs)], it generally grouped with other lower Rhine populations in trees and individual assignment results. The Schanzengraben population consistently showed low genetic diversity and heterozygosity compared to other populations, including the extinct Sense population, and the individuals sampled for this study fell into a restricted range of age classes $(3+$ to $7+)$. This population also harboured very little unique genetic diversity when compared to the lower Rhine cluster as a whole. While eleven of the 22 individuals sampled within this population had a high likelihood of being full-sibs, this is unlikely to be due to biased sampling as the methods and effort employed in sampling this population were the same as in other populations; it is therefore likely that the low genetic diversity and high relatedness of this sample merely reflects the overall individual makeup of the Schanzengraben population. Several dams have been built on the lower Limmat River that may prevent nase migration from the Rhine to the Schanzengraben, reducing gene flow between this nase population and others, contributing to further genetic isolation through drift. Thus it seems likely that the Schanzengraben population has been through a severe bottleneck with little subsequent gene flow with other nase populations, resulting in the present low levels of genetic diversity. This bottleneck will have had a profound effect on the population's allele frequencies that in turn would have strongly contributed to the genetic distinctness of this population, more so than the potential evolution/retention of unique intraspecific genetic diversity.

In this study, the maximum number of mutational steps found among individual mitochondrial haplotypes was only six, with no clear signal of historical isolation and divergence (i.e. multiple, missing intermediate haplotypes). This suggests that Swiss nase populations and potentially all Western and Central European populations are derived from colonizers from a single glacial refugium. The higher diversity of mtDNA haplotypes in the Danubian sample, coupled with the restricted geographic distribution of nase in Western Europe to tributaries of the Rhine, relative to its range in Eastern Europe, indicate that this refugium was 
located within the Danube catchment. Whilst most other European temperate freshwater fish species show clear mtDNA phylogeographic signals of a Danubian refugium, the majority of these species also show higher levels of intraspecific haplotypic diversity, both within and among populations (Hewitt 2004; Costedoat and Gilles 2009). One species that shows similar patterns to the nase of low mtDNA lineage diversity in Central Europe, is the European barbel (Barbus barbus) (Kotlík and Berrebi 2001). This cyprinid is also rheophilic and requires heterogeneous, interconnected riverine habitats to thrive (Britton and Pegg 2011). Overall, for European freshwater fish faunas, riverine specialists show marked latitudinal declines in species richness towards formerly glaciated regions (Griffiths 2006). Therefore the low levels of mitochondrial haplotype diversity found in this study likely indicate that the specialized ecology of the nase, involving spawning migrations and ontogenetic shifts between disjunct feeding niches, have left the nase especially vulnerable to environmental fluctuations, both during the climactic turmoil of the Quaternary ice ages as well as in more recent times due to anthropogenic impacts.

Evidence for local adaptation and implications for conservation management

One of the key criteria in assigning conservation priorities at the intraspecific level is the degree of ecological exchangeability between major population units (Crandall et al. 2000). By measuring intraspecific adaptive differentiation within a species and conserving distinct population units based on this criterion, this will preserve adaptive genetic diversity within the species and help to maximise its future evolutionary potential. Measured levels of adaptive divergence among identified population units can also be used to inform conservation management practices such as genetic rescue and reintroduction schemes, helping to avoid the potential pitfalls of outbreeding depression (Crandall et al. 2000; Fraser and Bernatchez 2001; Funk et al. 2012). In this study, to survey levels of adaptive differentiation among populations of Swiss nase, we employed a 'phenotypedown/genotype-up' approach. Genomic evidence for local adaptation came from comparing the relative numbers of $F_{\mathrm{ST}}$ outlier loci detected within different population comparisons. Within the lower Rhine cluster, genome scans identified one candidate locus for evolving under divergent natural selection: TCTA-318. The presence allele at this locus had a lower prevalence $(33.33 \%)$ in the Schanzengraben population, compared to other lower Rhine populations ( 81.25 to $100 \%$ ), suggesting that this population may have adapted to local selection regimes. However, the selection candidate status for this locus was only supported by the DFDIST analysis and only for one of the two multitest correction methods employed. No further significant $F_{\mathrm{ST}}$ outlier loci were found in this study, whether between populations in the same genetic cluster or in pairwise comparisons between populations from the different lower Rhine and Lake Constance genetic clusters. The results of these genome scans could be interpreted as divergent natural selection having had limited impact on shaping patterns of adaptive genetic diversity among the constituent populations of the Swiss nase. However, given a maximum number of 377 AFLP loci for a given outlier scan data set, we sampled the genome at an average marker density of one marker per $>2 \mathrm{Mb}$ (genome size $1.4 \mathrm{~Gb}$; www.genomesize. com), meaning that only very large regions of genomic differentiation would likely be detected. Further power limitations to detect loci evolving under divergent selection involve the limited number of individuals genotyped per population (six to 17). This lack of power is best illustrated by outlier scan comparisons between paired populations from the lower Rhine and Constance clusters, where the strongest candidate locus (GGTA-257) had presence allele frequencies of 71.43 to $81.25 \%$ in the Lake Constance populations compared to frequencies of 0 to $12.5 \%$ in the lower Rhine populations. Despite these large allele frequency differences, this locus was not identified as a selection candidate locus in any of the pairwise population comparisons, once multitest correction had been carried out (in eleven out of fourteen DFDIST comparisons, this locus had $F_{\text {ST }}$ values above the 0.95 quantile). Whilst our study design certainly lacks the power to identify all genomic regions evolving under divergent natural selection, other intraspecific studies utilising similar numbers of loci and individuals in their data sets have found multiple $F_{\mathrm{ST}}$ outliers that are strong candidates for loci evolving under divergent natural selection (e.g. Wilding et al. 2001; Bonin et al. 2006; Nosil et al. 2008; Keller et al. 2012; Hudson et al. 2013). Thus, although our genome scans cannot rule out some level of local adaptation, the lack of strong $F_{\mathrm{ST}}$ outliers, coupled with generally non-significant genetic differentiation among Swiss nase populations within the same principal genetic clusters, suggests that the genetic signature of local adaptation within population clusters may not be widespread in the genome.

Although hierarchical structuring of genetic variation amongst populations was explicitly taken into account whilst running outlier analyses, other demographic factors that may cause biases in the outlier detection process could not be ruled out (Excoffier et al. 2009). Historical population events such as bottlenecks could violate demographic models employed by both BAYESCAN and DFDIST, potentially resulting in higher rates of false negatives/positives amongst the candidate loci identified (Butlin 2010). This problem is not unique to our study and will affect the implementation of outlier detection methodologies in many conservation 
genetic studies. Further research is therefore needed to ascertain the impact of population decline on the neutral variance in the $F_{\mathrm{ST}}$ distribution and its implications for identifying divergent selection candidate loci in studies of local adaptation (Funk et al. 2012). TCTA-318 was the only significant $F_{\mathrm{ST}}$ outlier locus identified in this study and is, by default, the most realistic candidate for either being, or closely linked to, a target locus for divergent selection regimes. Although any full-sib pairs identified in the Schanzengraben population were removed from the outlier scan data set, closely related individuals (e.g. half-sibs) could still predominate among the remaining individuals of this population data set, potentially contributing to the changes in allele frequencies seen at this locus; this could result in TCTA-318 being wrongly identified as a candidate locus. The issue of false positives and what constitutes a real selection candidate locus is inherent to all population genomic studies and further research is needed to sequence and characterize the genomic regions in question (Butlin 2010).

To complement the genomic data, phenotypic evidence for the existence of local adaptation among Swiss nase populations came from the geometric morphometric analysis of body shape divergence among Swiss nase populations. Two major axes of population morphological differentiation were found: (i) differences in body shape between the Schanzengraben population and most other populations sampled (CV 1 axis), and (ii) between populations spawning in the Wiese and Birs and those spawning in the Murg, Rotbach and Binnenkanäle ( $\mathrm{CV}$ axis 2). However, no significant association between AFLP loci and GM aspects of body shape was found in logistic regression analyses. The lack of significant association between marker loci and body shape could be due to the relatively limited genomic resolution offered by the number of loci employed in the genome scans (see above). Also the phenotypic components that make up overall body shape almost certainly have a polygenic architecture, rather than being due to major allele frequency changes at one or a few loci. Therefore, changes in the genetic loci underlying these traits could be largely invisible to $F_{\mathrm{ST}}$ outlier detection methods (Le Corre and Kremer 2012). Nase body shape may also comprise a major non-heritable component, with plasticity playing a role in population-specific phenotypic divergence; although levels of phenotypic plasticity in adaptive traits may be population-specific, evolving in response to local selection regimes (West-Eberhard 2003). Therefore more research, both genetic and ecological, needs to be carried out to ascertain whether local divergence in nase body shape could represent adaptive divergence to distinct riverine habitats.

In light of the results of this study, we recommend that the Swiss lower Rhine and Lake Constance population groupings be managed as separate CUs within $C$. nasus (Fraser and Bernatchez 2001; Funk et al. 2012). Whilst more research needs to be undertaken to measure levels of divergent adaptation between these distinct CUs, levels of genetic divergence between the Lake Constance, the lower Rhine and the Danube imply they have been following independent evolutionary trajectories for hundreds, if not thousands, of generations (Fraser and Bernatchez 2001). Therefore, individuals chosen for any reintroduction/translocation projects should be sourced from demographically healthy populations within the appropriate CU. Indeed one prime candidate for such a reintroduction program would be the Sense River, whose indigenous nase population has now been extirpated and was found in this study to group firmly with other extant nase populations within the lower Rhine cluster. We note again the confirmed presence of an adult individual of the lower Rhine genotype cluster sampled from one tributary to Lake Constance that is dominated by individuals bearing the Lake Constance cluster genotypes. Nase from the Murg River were stocked into rivers flowing into Lake Constance from 1996 to 1998 to supplement declining local populations (M. Kugler pers. comm.). This highlights one of the potential pitfalls of implementing conservation management projects without detailed knowledge of the geographic structuring of intraspecific diversity. Indeed, future research should be carried out to address the intriguing question of the evolutionary origin of the distinct Lake Constance nase, with particular focus on nase populations from German or Austrian rivers draining into Lake Constance not included in this study. One possible exception to the above conservation strategy of sourcing individuals from the same CU for supplemental introductions is the distinct Schanzengraben population. Genetic differentiation of this population may not solely be due to genetic drift and inbreeding within a small population, as this study has revealed population genomic and phenotypic evidence for local adaptation. More research needs to be carried out on the Schanzengraben population to resolve the interplay between genetic drift and selection and the implications this has for the long-term persistence and adaptive potential of this population. We suggest that efforts be made to manage this population as a subhierarchical CU within the overall lower Rhine CU, thus preserving potential unique genetic and ecological diversity. In general, we recommend that management plans be put in action to first improve the quality and connectivity of the juvenile and adult nase habitats, followed by monitoring of population viability, before any attempts at genetic rescue/ restoration of Swiss nase populations be made.

Acknowledgments We kindly acknowledge for providing samples: Dr. Arthur Kirchhofer for historical Sense samples, Dr. Wolfgang Mark for Danubian samples, Franck Bonell for Dornbirner Ach samples, Dr. Caroline Costedoat for P. toxosoma samples. We thank Dr. Michel Dedual, Franck Bonell for performing the aging on scales. 
We thank Salome Mwaiko, Isabel Magalhaes, Andreas Taverna, Guy Périat, Brigitte Germann, Denise Weibel, Sabina Käppeli, Bänz Lundsgaard Hansen, Denis Roy, Kay Lucek, Andreas Hertig, Rachel Tucker, Markus Grünenfelder, Martin Huber Gysi, Daniel Zopfi, Fredi Fehr, Michael Kugler for their assistance. We thank WWF Switzerland for making this study possible. We acknowledge finan cial support by the Federal Office for the Environment (FOEN).

\section{References}

Adamik Z, Obrdlik P (1977) Food of important cyprinid species in the warmed barb zone of the Oslava River. Folia Zool 25:171 182

Allendorf FW, Hohenlohe PA, Luikart G (2010) Genomics and the future of conservation genetics. Nat Rev Genet 11:697 709

Ashley MV, Willson MF, Pergams ORW, O'Dowd DJ, Gende SM, Brown JS (2003) Evolutionarily enlightened management. Biol Conserv 111:115 123

Barluenga M, Sanetra M, Meyer M (2006) Genetic admixture of burbot (Teleostei: lota lota) in Lake Constance from two European glacial refugia. Mol Ecol 15:3583 3600

Beaumont MA, Balding DJ (2004) Identifying adaptive genetic divergence among populations from genome scans. Mol Ecol 13:969 980

Behrmann Godel J, Gerlach G, Eckmann R (2004) Postglacial colonization shows evidence for sympatric population splitting of Eurasian perch (Perca fluviatilis L.) in Lake Constance. Mol Ecol 13:491 497

Benjamini Y, Hochberg Y (1995) Controlling the false discovery rate: a practical and powerful approach to multiple testing. J R Stat Soc 57:289 300

Bernatchez L (2001) The evolutionary history of brown trout (Salmo trutta L.) inferred from phylogeographic, nested clade, and mismatch analyses of mitochondrial DNA variation. Evolution $55: 351379$

Bernatchez L, Wilson CC (1998) Comparative phylogeography of Nearctic and Palearctic fishes. Mol Ecol 7:431 452

Bonin A, Bellemain E, Eidesen PB, Pompanon F, Brochmann C, Taberlet $\mathrm{P}$ (2004) How to track and assess genotyping errors in population genetics studies. Mol Ecol 13:3261 3273

Bonin A, Taberlet P, Miaud C, Pompanon F (2006) Explorative genome scan to detect candidate loci for adaptation along a gradient of altitude in the common frog (Rana temporaria). Mol Biol Evol 23:773 783

Bookstein FL (1991) Morphometric tools for landmark data, geom etry and biology. Cambridge University Press, Cambridge

Bouzat JL (2010) Conservation genetics of population bottlenecks: the role of chance, selection, and history. Conserv Genet 11:463 478

Britton JR, Pegg J (2011) Ecology of European Barbel Barbus Barbus: implications for river, fishery, and conservation man agement. Rev Fish Sci 19:321 330

Brunner PC, Douglas MR, Osinov A, Wilson CC, Bernatchez L (2001) Holarctic phylogeography of arctic charr (Salvelinus alpinus L.) inferred from mitochondrial DNA sequences. Evolution 55:573 586

Butlin RK (2010) Population genomics and speciation. Genetica 138:409 418

Caravajal Rodriguez A, de Una Alvarez J, Rolan Alvarez E (2009) A new multitest correction (SGoF) that increases its statistical power when increasing the number of tests. BMC Bioinf 10:209

Cornuet JM, Luikart G (1997) Description and power analysis of two tests for detecting recent population bottlenecks from allele frequency data. Genetics 144:2001 2014
Costedoat C, Gilles A (2009) Quaternary pattern of freshwater fishes in Europe: comparative phylogeography and conservation per spective. Open Conserv Biol J 3:36 48

Crandall KA, Bininda Emonds ORP, Mace GM, Wayne RK (2000) Considering evolutionary processes in conservation biology. Trends Ecol Evol 15:290 295

Dedual M (1990) Biologie et problèmes de dynamique de population du nase (Chondrostoma nasus nasus) dans la petite Sarine. PhD thesis, Université de Fribourg

Di Rienzo A, Peterson AC, Garza JC, Valdes AM, Slatkin M, Freimer NB (1994) Mutational processes of simple sequence repeat loci in human populations. Proc Natl Acad Sci USA 91:3166 3170

Durand JD, Persat H, Bouvet Y (1999) Phylogeography and postglacial dispersion of the chub (Leuciscus cephalus) in Europe. Mol Ecol 8:989 997

Edmands S (2007) Between a rock and a hard place: evaluating the relative risks of inbreeding and outbreeding for conservation and management. Mol Ecol 16:463 475

Englbrecht CC, Freyhof J, Nolte A, Rassmann K, Schliewen U, Tautz D (2000) Phylogeography of the bullhead Cottus gobio (Pisces: Teleostei: Cottidae) suggests a pre Pleistocene origin of the major central European populations. Mol Ecol 9:709 722

Excoffier L, Lischer HEL (2010) Arlequin suite ver 3.5: a new series of programs to perform population genetics analyses under Linux and Windows. Mol Ecol Resour 10:564 567

Excoffier L, Hofer T, Foll M (2009) Detecting loci under selection in a hierarchically structured population. Hereditary 103:285 298

Felsenstein J (1989) PHYLIP phylogeny inference package (Ver sion 3.2). Cladistics 5:164 166

Foll M, Gaggiotti O (2008) A genome scan method to identify selected loci appropriate for both dominant and codominant markers: a bayesian perspective. Genetics 180:977 993

Frankham R, Ballou JD, Eldridge MDB, Lacy RC, Ralls K, Dudash MR, Fenster CB (2011) Predicting the probability of outbreeding depression. Conserv Biol 25:465 475

Fraser DJ, Bernatchez L (2001) Adaptive evolutionary conservation: towards a unified concept for defining conservation units. Mol Ecol 10:2741 2752

Funk CF, McKay JK, Hohenlohe PA, Allendorf FW (2012) Harnessing genomics for delineating conservation units. Trends Ecol Evol 27:489 496

Gollmann G, Bouvety Y, Karakousis Y, Triantaphyllidis C (1997) Genetic variability in Chondrostoma from Austrian, French and Greek rivers (Teleostei, Cyprinidae). J Zool Syst Evol Res 35:165 169

Goudet J (1999) PCA GEN (version 1.2), a computer package which performs principal component analysis (PCA) on gene frequency data. Available from http://www.unil.ch/izea/softwares/pcagen. html

Goudet J (2001) FSTAT, a program to estimate and test gene diversities and fixation indices (version 2.9.3). http://www.unil. $\mathrm{ch} /$ izea/softwares/fstat.html

Griffiths G (2006) Pattern and process in the ecological biogeography of European freshwater fish. J Anim Ecol 75:734 751

Guo SW, Thompson EA (1992) Performing the exact test of Hardy Weinberg proportion for multiple alleles. Biometrics 48:361 372

Hedrick PW, Fredrickson R (2010) Genetic rescue guidelines with examples from Mexican wolves and Florida panthers. Conserv Genet 11:615 626

Hewitt GM (2004) Genetic consequences of climatic oscillations in the Quaternary. Phil Trans R Soc B 359:183 195

Holsinger KE, Lewis PO (2007) Hickory, software for analysis of geographic structure in genetic data. http://darwin.eeb.uconn. edu/hickory/hickory.html 
Huber Gysi M (2009) Die Bestandssituation der Nase Chondrostoma nasus in der Schweiz 2005 2008. WWF, Switzerland

Huber M, Kirchhofer A (1998) Radio telemetry as a tool to study habitat use of nase (Chondrostoma nasus L.) in medium sized rivers. Hydrobiologia 371(372):309 319

Huber M, Kirchhofer A (2001) Reproductive success of nase (Chondrostoma nasus L.) and its influence on population dynamics. Arch Hydrobiol Suppl 135:307 330

Hudson AG, Vonlanthen P, Seehausen O (2011) Rapid parallel adaptive radiations from a single hybridogenic ancestral popu lation. Proc R Soc B 278:58 66

Hudson AG, Vonlanthen P, Bezault E, Seehausen O (2013) Genomic signatures of relaxed disruptive selection associated with speci ation reversal in whitefish. BMC Evol Biol 13:108

Hughes JB, Daily GC, Ehrlich PH (1997) Population diversity: its extent and extinction. Science 278:692 698

Jones OR, Wang J (2010) COLONY: a program for parentage and sibship inference from multilocus genotype data. Mol Ecol Resour 10:551 555

Joost S, Bonin A, Bruford MW, Despres L, Conord C, Erhardt G, Taberlet P (2007) A spatial analysis method (SAM) to detect candidate loci for selection: towards a landscape genomics approach to adaptation. Mol Ecol 16:3955 3969

Jump AS, Hunt JM, Martinez Izquierdo JA, Penuelas J (2006) Natural selection and climate change: temperature linked spatial and temporal trends in gene frequency in Fagus sylvatica. Mol Ecol 15:3469 3480

Junker J, Armin P, Wagner CE, Mwaiko S, Germann B, Seehausen O, Keller I (2012) River fragmentation increases localized popula tion genetic structure and enhances asymmetry of dispersal in bullhead (Cottus gobio). Conserv Genet 13:545 556

Keller I, Schuler J, Bezault E, Seehausen O (2012) Parallel divergent adaptation along replicated altitudinal gradients in Alpine trout. BMC Evol Biol 12:210

Kirchhofer A (1996) Fish conservation in Switzerland three case studies. In: Kirchhofer A, Hefti D (eds) Conservation of endangered freshwater fish in Europe. Birkhäuser Verlag, Basel, pp 135145

Klingenberg CP (2011) MorphoJ: an integrated software package for geometric morphometrics. Mol Ecol Resour 11:353 357

Kohn MH, Murphy WJ, Ostrander EA, Wayne RK (2006) Genomics and conservation genetics. Trends Ecol Evol 21:629 637

Kotlík P, Berrebi P (2001) Phylogeography of the barbel (Barbus barbus) assessed by mitochondrial DNA variation. Mol Ecol 10:2177 2185

Kottelat M, Freyhof J (2007) Handbook of European freshwater fishes. Kottelat, Cornol and Freyhof, Berlin

Kristensen TN, Pedersen KS, Vermeulen CJ, Loeschcke V (2010) Research on inbreeding in the 'omic' era. Trends Ecol Evol $25: 4452$

Le Corre V, Kremer A (2012) The genetic differentiation at quantitative trait loci under local adaptation. Mol Ecol 21:1548 1566

Mace GM, Purvis A (2008) Evolutionary biology and practical conservation: bridging a widening gap. Mol Ecol 17:9 19

Maier KJ, Zeh M, Ortlepp J, Hefti D (1995) Mitteilungen zur fischerei nr. 53: Verbreitung und Fortpflanzung der in der Schweiz vorkommenden Chondrostoma Arten. Bundesamt für Umwelt, Bern

Mäkinen HS, Merilä J (2008) Mitochondrial DNA phylogeography of the three spined stickleback (Gasterosteus aculeatus) in Eur ope evidence for multiple glacial refugia. Mol Phylogenet Evol 46:167 182

Mesquita N, Cunha C, Hanfling B, Carvalho GR, Ze Ze L, Tenreiro $\mathrm{R}$, Coelho MM (2003) Isolation and characterization of polymorphic microsatellite loci in the endangered Portuguese freshwater fish Squalius aradensis (Cyprinidae). Mol Ecol Notes 3:572 574

Muenzel FM, Sanetra M, Salzburger W, Meyer A (2007) Microsat ellites from the vairone Leuciscus souffia (Pisces: Cyprinidae) and their application to closely related species. Mol Ecol Notes $7: 10481050$

Nesbø CLT, Fossheim L, Vøllestad A, Jakobsen KS (1999) Genetic divergence and phylogeographic relationships among European perch (Perca fluviatilis) populations reflect recent glacial refugia and postglacial colonization. Mol Ecol 8:1387 1404

Nosil P, Egan SP, Funk DJ (2008) Heterogeneous genomic differ entiation between walking stick ecotypes: "isolation by adapta tion" and multiple roles for divergent selection. Evol 62:316 336

Ouborg NJ, Pertoldi C, Loeschcke V, Bijlsma R, Hedrick PW (2010) Conservation genetics in transition to conservation genomics. Trends Genet 26:177 187

Peňáz M (1996) Chondrostoma nasus its reproduction strategy and possible reasons for a widely observed population decline a review. In: Kirchhofer A, Hefti D (eds) Conservation of endangered freshwater fish in Europe. Birkhäuser Verlag, Basel, pp 279285

Pérez Figueroa A, Saura M, Fernández J, Toro MA, Caballero A (2009) METAPOP A software for the management and analysis of subdivided populations in conservation programs. Conserv Genet 10:1097 1099

Pritchard JK, Stephens M, Donnelly P (2000) Inference of population structure using multilocus genotype data. Genetics 155:945 959

Rakowitz G, Berger B, Kubecka J, Keckeis H (2008) Functional role of environmental stimuli for the spawning migration in Danube nase Chondrostoma nasus L. Ecol Freshw Fish 17:502 514

Raymond M, Rousset F (1995) Genepop (Version 1.2) population genetics software for exact tests and ecumenicism. J Hered 86:248 249

Reckendorfer W, Keckeis H, Tiitu G, Winkler G, Zornig H, Schiemer F (2001) Diet shifts in 0+ nase, Chondrostoma nasus: size specific differences and the effect of food availability. Arch Hydrobiol Suppl 135:425 440

Rice WR (1989) Analyzing tables of statistical tests. Evolution 43:223 225

Rohlf FJ (2006) TPSDig Version 2.1. State University of New York, Stony Brook

Salzburger W, Brandstätter A, Gilles A, Parson W, Hempel M, Sturmbauer C, Meyer A (2003) Phylogeography of the varione (Leuciscus souffia, Risso 1826) in Central Europe. Mol Ecol 12:2371 2386

Schluter D (1996) Ecological speciation in postglacial fishes. Phil Trans R Soc B 351:807 814

Stockwell CA, Hendry AP, Kinnison MT (2003) Contemporary evolution meets conservation biology. Trends Ecol Evol 18:94 101

Szabo Z (1958) Beiträge zur Vermehrungsbiologie der Nase (C. nasus L.). Z Fisch N F 7:631 636

Taylor EB (1999) Species pairs of north temperate freshwater fishes: evolution, taxonomy, and conservation. Rev Fish Biol Fish 9:299 324

Thornton KR, Jensen JD (2007) Controlling the false positive rate in multilocus genome scans for selection. Genetics 175:737 750

Vekemans X (2002) AFLP SURV version 1.0. Laboratoire de Génétique et Ecologie Végétale, Université Libre de Bruxelles, Belgium

Vonlanthen P, Excoffier L, Bittner D, Persat H, Neuenschwander S, Largiadèr CR (2007) Genetic analysis of potential postglacial watershed crossings in Central Europe by the bullhead (Cottus gobio L.). Mol Ecol 16:4572 4584 
Vos P, Hogers R, Bleeker M, Reijans M, Vandelee T, Hornes M, Frijters A, Pot J, Peleman J, Kuiper M, Zabeau M (1995) AFLP a new technique for DNA fingerprinting. Nucleic Acids Res 23:4407 4414

Vyskocilova M, Simkova A, Martin JF (2007) Isolation and characterization of microsatellites in Leuciscus cephalus (Сyp riniformes, Cyprinidae) and cross species amplification within the family Cyprinidae. Mol Ecol Notes 7:1150 1154

Weeks AR, Sgro CM, Young AG et al (2011) Assessing the benefits and risks of translocations in changing environments: a genetic perspective. Evol Appl 4:709 725
Weir BS, Cockerham CC (1984) Estimating $F$ statistics for the analysis of population structure. Evolution 38:1358 1370

West Eberhard MJ (2003) Developmental plasticity and evolution. Oxford University Press, Oxford

Wilding CS, Butlin RK, Grahame J (2001) Differential gene exchange between parapatric morphs of Littorina saxatilis detected using AFLP markers. J Evol Biol 14:611 619

Willi Y, Van Buskirk J, Hoffmann AA (2006) Limits to the adaptive potential of small populations. Annu Rev Ecol Evol Syst $37: 433458$ 